\title{
On the Multi-dimensional Relationship of Visual Symbols in Film Posters
}

\author{
Yuying Tao ${ }^{1, *}$
}

\author{
${ }^{1}$ College of Fine Arts and Design, Jimei University \\ *Corresponding author.Email:865780976@qq.com
}

\begin{abstract}
Film poster is a visual image that publicizes and expounds the film plot. The unique visual symbols in its image design can arouse the empathy of the audience. The significance of its visual image has changed from the ideographic meaning of publicity to the deep visual culture and structure. From the perspective of viewers and creators, based on visual culture, disassemble the visual symbols of film posters according to the needs of subject, spatial dimension, color, emotion and technology, intuitively understand the multi-dimensional relationship, development process and characteristics of visual symbols in film posters, and analyze the graphic thinking and development trend of film posters in the digital environment.
\end{abstract}

Keywords: visual symbols, movie posters, multi-dimensional relationship

\section{INTRODUCTION}

Saussure said: "The combination of concept and audiovisual, I call it a symbol." Movies are a major development trend of digital design. With the advancement of digital technology, visual images obtained by using digital images to express "borderless" thinking and concrete space through imagining scenarios are called the concept of contextualized design. This concept highlights used in movie concept poster design and special effects lens application. Modern symbols are widely used in the design of concept posters and lenses in special effects movies. The purpose and significance is to reflect their visual elements and reproduce their thinking system through digital technology, and to transform digital movie design from static and synchronic to open, dynamic, and diachronic transformation. This paper describes the visual culture and image interpretation of digital lens and conceptual poster design in special effects movies, and shows how the multi-dimensional relationship of visual symbols in movie posters is embodied in the concept of movie situational design.

\section{FILM VISUAL DESIGN CLASSIFICATION AND OVERALL CHARACTERISTICS}

\subsection{STATIC POSTER AND MOTION CAMERA DESIGN IN FILM}

Film is the most basic image file, which is divided into basic categories based on the different image narratives presented by different technologies. Based on the commonality of movies, films can be divided into different types, such as women's films, children's films, war films, romance films, comedy films, tragic films, biopics and so on[1]. In the lens design of movies, visual design will be classified according to the classification of film types, such as lens application and lens Angle. The use of shots in movies is principally to portray the psychology and narrative process of characters. Visual elements such as field, angle, light and color are predominantly used in the design of shots. For motion shot, their rhythm, emotional depiction and characterization of characters pave the way for movie narration.

Static posters in movies are an important visual element that encapsulates the story. A motion shot consists of stationary images, and each frame in the shot is unique and indispensable. The visual logic in the motion shot can better reveal the content of the story. 
What is seen and gained visually is reflected in the form of symbols, patterns, sounds, colors and sizes. Only by fixing the visual symbols in the "moving" lens in the "static" visual image and placing the symbols of the external world in the individual's internal world can the dynamic and static transformation in visual design have significance. It is the summary and induction of visual design in movies to plan the visual elements of dynamic shots into static images and cover and tell the story in a brief language.

\subsection{The general characteristics of digital design in film}

Digital design is not only used in art related fields, computer graphics are widely used in image design in journalism, movies and other fields. Movies use digital technology to create visual images, and show "borderless" thinking through the application of visual symbols in images. This concept is prominently reflected in the concept poster design and special effects shots of movies. Modern symbols are widely used in the design of concept posters and shots in movies, whose purpose and significance lies in the multi-directional and multi-angle embodiment of visual symbols by digital technology. [2]

Digital design in the Internet era can be divided into digital painting, 3D modeling and the development of network transmission technology. Breaking stereotypes is the basic thinking of digital design into image thinking. The transformation of digital design from twodimensional to three-dimensional makes its application in movies bold, open, more popular and intuitive. Digital technology opens a new path in the field of film production, and solves problems such as spatial integration and the practicability of virtual characters with digital technology[3]. Digital technology has closely linked the relationship between virtual characters and real actors, making the body language, facial expressions and psychological changes of movie characters similar to those of real actors, replacing the necessary dangerous movements of actors in movies. Virtual shooting and virtual real shooting are obtained from virtual production technology, and the nearly real movie scenes and motion shots are shaped in an allround way. In addition, traditional thinking is broken to some extent, new CG scenes are constructed, and new design ideas and creative content are given to film visual design.

The American film industry, which is famous for its technology, subverts the "sense of real existence" of traditional films by applying visual elements in film shots or posters with more $3 \mathrm{D}$ simulation technology to bring an immersive experience to people. The development of the American film industry to a certain extent promotes the application of digital technology in the film industry. Software such as AE and 3Dmax, as the leading role in the post-production of movies, promotes the output of digital films. The realistic and thrilling visual feelings brought by $3 \mathrm{D}$ movies bring huge creative space for creators. 3D technology brings creators an important expression of "deep space", which places visual symbols in cinema and television space according to form and narrative logic, bringing challenges and opportunities to visual creation in films. From a higher perspective, the problem of harmony between $3 \mathrm{D}$ visual symbols and original symbols in films has not been solved. The audio-visual aesthetics developed in movies for a long time is restricted by $3 \mathrm{D}$ technology, and the tale itself is commonly separated by the presentation technology in 3D immersive experience. But on the other hand, digital 3D technology promotes the innovation of visual thinking in movies, enriches the content of visual re-creation, and makes the visual images in movies bring people great immersive experience and novelty. The combination of audio-visual language and technology will once again promote the development of visual art in films.

\section{THE EMBODIMENT OF THE VISUAL SYMBOL "MULTI-DIMENSIONAL RELATION" IN THE SITUATIONAL CONCEPT DESIGN OF FILM}

\subsection{The concept of Situational Design in film design}

As a powerful means of expressing abstract objects such as thinking space and emotion, image technology can most effectively express its connotation under a certain degree of situational design. Situational design is an art design technique based on the extension of digital creation. Its connotation is to show the specific situation and thinking through digital means, and use the unique visual elements in the situation to arouse people's psychological and spiritual emotional resonance.

Movie posters show the time, place and characters of the story through traditional design means such as painting and photography. However, the development of technology in the image age makes the Situational Design break through the traditional form and create a "nothingness" visual image. Among them, the conceptual design of film posters has gradually changed from "rational" design to "borderless" irrationality. In the concept of situational design, the concrete setting of thinking scene and the abstraction of time visual elements are integrated into the scene design or displayed through character elements. In the design of visual symbols, in addition to the intuitive, specific and attractive "Scene", it is also necessary to conceive the factors and multi-dimensional relationship of its composition and meaning in the scene. 
Visual symbols are embodied through "scenery", and their thinking space is embodied through "environment". Situational interpretation is also image interpretation. Formal analysis and image interpretation are effective tools to understand the image world[4]. Image interpretation is tortuous. No matter which level to analyze, once the perspective of the viewer is substituted, the meaning of the image and the viewer will change. The situation is closely related to the spiritual level. Chinese landscape painting mostly uses ink to express artistic conception. Traditional modeling pays attention to creating artistic conception, and the form and spirit have. When the artistic conception is brought into the visual image scene of the film, the special visual symbols will bring different context and visual experience.

Taking the film White Snake as an example, the movie poster is mainly based on virtual scenes, and uses two visual symbols: low saturation color and exaggerated sea water to shape the repressive atmosphere in the film. First, it explains the background of the story, but renders the protagonist's complex psychology. Behind the "scenery", the visual implication of "environment" in Chinese landscape painting is the visual element that brings imagination space to the visual image. Its composition, color and emotional arrangement will arouse our unique emotional resonance. This is not only a special visual art symbol, but also an aesthetic emotion, detached from real space, so that we can feel the spiritual world rather than a means. The thought-provoking ink painting style puts Leifeng Tower and male and female protagonists in a diagonal position, and abstracts the main body and secondary body through black-and-white contrast, which is thought-provoking.

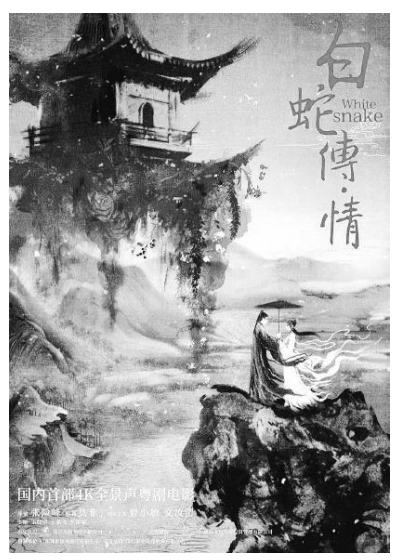

Figure 1 Movie White Snake ink painting Poster

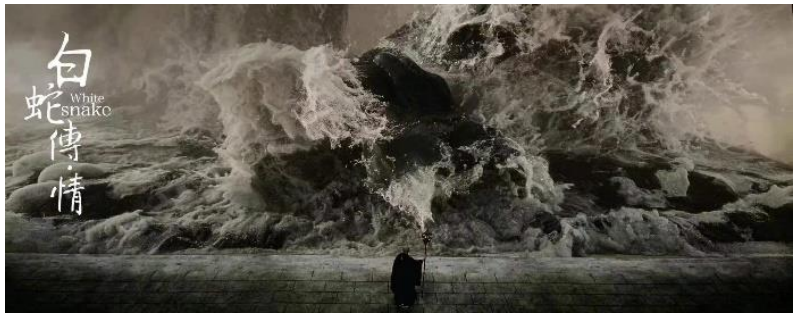

Figure 2 Movie White Snake Poster

\subsection{The embodiment of visual symbol "multi- dimensional relationship"}

Visual symbols are gradually changing with the development of civilization. At the beginning, visual symbols are simple and plain, and their main meaning is to record events. In the initial stage, humans ' demand for visual symbols is low, and their forms and styles are monotonous[5]. Its development trend is also from simple line patterns to painting art progress, from memory to showing the spiritual world. With the progress of human civilization and technology in social development, society pays more and more attention to the presentation of images. The replication of visual images is more and more rapid, and the scope of visual art is gradually increasing. Visual symbols have changed from single to diversified, mainly through lines, colors, composition and other visible ways to show, is the expression of design ideas and connotations of symbols, mainly used to convey a variety of information. At the beginning, visual symbols were endowed with the meaning of "combination of concept and audio and video". With the development of The Times, visual symbols gradually separated from the scope of "signifier" and "signified", and merged with the multi-directional content extended by society, and were used in more visual image design through different forms, different contents and different meanings.

Nowadays, in the era of images, people's visual needs and images of beauty have changed, and visual memory has developed from single to diversified. Visual symbols in film images are combined with literature, technology and psychology, and also produce different developments in cross-field fusion. Image and language, image and psychological, images and text, image and new media art, image and new technology, image and information, image and narrative, image and aesthetic, the combination of image and functions such as image, the visual symbol was differentiated into language symbols, psychological symbol, text symbols, symbols of technology and information symbol, function symbols. Language symbols promote the dissemination of information, which promotes the development of writing. Multi-dimensional relations promote the birth of a new perspective, which corresponds to human psychology and thinking. 
The visual image in the film is more simplified and generalized the multi-dimension of visual symbols. An image covers psychological design, scene design, color design, language design and image narrative design. Visual design and visual images serve people. Through technical deduction, visual design and multidisciplinary intersection extend the multi-perspective content and thought-provoking visual culture and narrative plot..[6]

\section{CONCLUSIONS}

Digital design promotes the thinking process of visual images in special effects movies, and visual thinking pushes back the sublimation of visual images, making the meaning of visual images not only summarize plots and display artistic concepts[7]. Visual symbols Under the collision of rationality and sensibility, the visual symbols in movie posters are endowed with more connotations, and they are more varied in terms of visual perception and thinking concept. These different forms and angles promote the occurrence of visual contextualization. Film is an art of modeling, which can not only fully highlight the advantages of three-dimensional space, but also deliberately show its own two-dimensional characteristics. Film creation requires dramatic conflicting narration and complex audio-visual language to organize the picture. Artists' experience and creative diversity can become the creation material of film themes, but paintings displayed on the screen will lose direction and boundaries and become endless imagination. Starting from the film itself, the creation background of the art work is rationally utilized, so that the work itself does not lose any characteristics, but has the spatial characteristics of the film, and becomes a part of the world that extends to all sides indefinitely [8]

The development order of modern art is that when art turns to self, modernism has its foundation, psychoanalysis begins to influence noumenon, and art begins to move towards self-discipline. Abstract art forms are born because of the trend towards artistic selfdiscipline. When artistic vision no longer describes stories, its form itself is the meaning and characteristics. The birth of photographic devices such as cameras replaced the narrative of artwork, visual images replaced language and text for narrative, and the selfdiscipline of language turned to society, and the art language was activated again. When the form moves towards extreme self-discipline, the exploration of human nature and self has infinite possibilities through the form. At present, the multi-way display of visual images is the multi-direction embodiment of visual symbols, and the multi-direction form of visual images promotes the multi-dimensional development of visual art. Contemporary visual art shifts the pattern of "painting", breaks fixed creation, and focuses on spiritual creation.

Through digital technology, visual art is not restricted by thinking and space in movie posters. The sense of historical space brought by it solves the function of reproducing reality. Digital optical images have more historical dimension and expression space, which is the second hand reality in the real sense and expresses the emotional value behind it.

In the context of the rapid development of visual culture, the application of visual symbols in the era of big data, image-based thinking in the era of reading pictures is of great significance. The development of contextualized design concept, through the embellishment of contextualized design concept in movie concept posters and special effects shots, all the tiny and lonely people can also give out their voices in digital scenes. The concept of situational design in the movie provides creative ideas for digital presentation, and visual symbols provide diversified perspectives for image development. It is no longer limited by creative means, but more in line with the freshness of visual images reflected in current social needs.

\section{REFERENCES}

[1] LAN fan A new theory of film types in 2012 Hundred artists, 28 (06): 133-144

[2] Song Wei. 2015 (01) the significance of visual symbols in the context of new media Beauty and the times (middle), 70-71

[3] Sheng Zhenyu 2020 (12) research on the virtual production era of digital technology film China mediatechnology,:122\124.DOI:10.19483/j.cnki.11 $-4653 /$ n.2020.12.035.

[4] Lu Hongyuan, Chen Hongyan, sun Qun 2020 (17) composition analysis in film and television posters Drama House: $86+96$

[5] Sui Nan 2018 explore the application of traditional symbols in Contemporary Chinese film and television posters Research on art education, (01): 93

[6] Li Xinchang 2017 (06) intertextual narration of images -- Taking film and television and film and television posters as an example Southern literary world,: 178-180

[7] $\mathrm{Hu}$ Huafang Image narration and aesthetic implication of 2021 movie posters Jiangxi Social Sciences, 41 (07): 240-246

[8] Yue Jiping, Wang Kun. 2021(21) The presentation of visual images and concepts of modern and contemporary art in films. Film Literature,:22-27. 